\title{
Lipolysis and lipases in white adipose tissue - An update
}

Andressa Bolsoni-Lopes', Maria Isabel C. Alonso-Vale²

\begin{abstract}
Lipolysis is defined as the sequential hydrolysis of triacylglycerol (TAG) stored in cell lipid droplets For many years, it was believed that hormone-sensitive lipase (HSL) and monoacylglycerol lipase (MGL) were the main enzymes catalyzing lipolysis in the white adipose tissue. Since the discovery of adipose triglyceride lipase (ATGL) in 2004, many studies were performed to investigate and characterize the actions of this lipase, as well as of other proteins and possible regulatory mechanisms involved, which reformulated the concept of lipolysis. Novel findings from these studies include the identification of lipolytic products as signaling molecules regulating important metabolic processes in many non-adipose tissues, unveiling a previously underestimated aspect of lipolysis. Thus, we present here an updated review of concepts and regulation of white adipocyte lipolysis with a special emphasis in its role in metabolism homeostasis and as a source of important signaling molecules. Arch Endocrinol Metab. 2015;59(4):335-42
\end{abstract}

Keywords

Adipose triglyceride lipase; hormone-sensitive lipase; monoacylglycerol lipase; perilipin; triacylglycerol hydrolysis
${ }^{1}$ Departamento de Fisiologia e Biofísica, Instituto de Ciências Biomédicas, Universidade de São Paulo (USP), São Paulo, SP, Brazil ${ }^{2}$ Departamento de Ciências Biológicas, Instituto de Ciências Ambientais, Químicas e

Farmacêuticas, Universidade Federal de São Paulo (Unifesp), Diadema, SP, Brazil

Correspondence to:

Maria Isabel C. Alonso Vale

Departamento de Ciências Biológicas Instituto de Ciências Ambientais, Químicas e Farmacêuticas,

Universidade Federal de São Paulo

Av. São Nicolau, 210

09913-030 - Diadema, SP, Brazil

alonsovale@gmail.com

Received on Mar/9/2015

Accepted on Apr/22/2015

DOI: 10.1590/2359-3997000000067

\section{INTRODUCTION}

$\mathrm{P}$ re-formed lipids that come from the diet or even those that are synthetized are stored mainly in the white adipose tissue (WAT) as triacylglycerols (TAG), neutral lipids made up of three fatty acids esterified to the carbon backbone of a glycerol molecule (1-3). The WAT, described as the main energy reservoir in mammals, has a mesenchymal origin and is made up by a matrix of connective tissue, immune system cells, bloods vessels, sympathetic innervation, stem cells and pre-adipocytes, as well as adipocytes, cells specialized in the intake, storage, and mobilization of fat (4).

Adipocytes store TAG in a single, large lipid droplet that takes up $85-90 \%$ of the cytoplasm and pushes the nucleus and a fine cytosol layer to the periphery of the cell. The lipid droplet is recognized as a dynamic cell organelle, surrounded by a monolayer of phospholipids, structural proteins, and enzymes, which protects cell organelles from the cytotoxic effects of fatty acids (5-7). Among the several proteins that make up this monolayer, perilipin IA may be emphasized, as it is the main perilipin found in the adipocyte. Depending on its activation status, perilipin $1 \mathrm{~A}$ may protect and delimit the lipid droplet, or make lipid hydrolysis easier $(7,8)$.

The amount of TAG stored in the adipocytes, which are in a constant flow in the TAG-fatty acid cycle, depends on the balance between lipogenesis (the biosynthesis, incorporation, and storage of TAG in the lipid droplet in the cytoplasm), and lipolysis (the sequential hydrolysis of TAG, fatty acids, and glycerol) $(1,9,10)$. In prolonged periods of positive energy balance, which result from excessive consumption of high-calorie foods added to the lack of physical activity, adiposity increases. This chronic process may lead to the development of obesity $(11,12)$. On the other hand, in prolonged periods of fasting or of high demands of energy, lipolysis is essential to supply fatty acids and glycerol as energy substrates to tissues. However, changes in this process have frequently been associated with lipodystrophy, hyperlipidemia, insulin resistance, and obesity $(12,13)$.

For many years, it was believed that hormone-sensitive lipase (HSL) and monoacyglycerol lipase (MGL) were the main enzymes involved in WAT lipolysis. However, after the discovery of the adipose triglyceride 
lipase (ATGL) in 2004 (14-16) and the later characterization of its actions and regulation mechanism, a new concept of lipolysis was proposed. During this period, evidence was found that indicated that, besides providing energy, final and intermediate products generated in the lipolysis process may act as signaling molecules that regulate important metabolic process in many non-adipose tissues, unveiling previously underestimated aspects of lipolysis. Therefore, we present here an updated review of lipolysis concepts and regulation in white adipocytes, with special emphasis in its role as a source of important signaling molecules in the control of metabolic homeostasis.

\section{LIPOLYSIS IN WHITE ADIPOCYTES}

Lipolysis consists of the sequential hydrolysis of TAG to its constituent molecules glycerol and three fatty acids, catalyzed by three different enzymes. In the first stage of this process, TAG is hydrolyzed to diacylglycerol (DAG) and one molecule of fatty acid, in a reaction mainly catalyzed by the enzyme ATGL. After that, DAG is converted to monoacylglycerol (MAG) and a second fatty acid by the action of HSL. In the end, MGL hydrolyses MAG, producing glycerol and a last fatty acid $(9,13)$ (Figure 1).

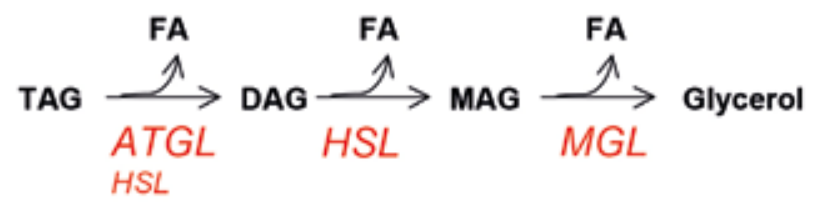

Figure 1. Sequential hydrolysis of triacylglycerol.

FA: fatty acid; ATGL: adipose triglyceride lipase; DAG: diacylglycerol; HSL: hormone-sensitive lipase; MGL: monoacylglycerol lipase; MAG: monoacylglycerol; TAG: triacylglycerol.

Fatty acids derived from lipolysis are, most of the times, released in the bloodstream and bound to albumin to be used as energy substrates by other tissues. However, part of these molecules is kept inside adipocytes, acting as intracellular signaling mediators and working as substrates for the synthesis of other fatty acids and lipids (including membrane lipids and other lipids involved in cell metabolism), as gene transcription regulators, oxidation substrates or undergoing reesterification to make up TAG again. Approximately $30-40 \%$ of these fatty acids undergo this reesterification, a process that is dependent on the intracellular generation of glycerol 3-phosphate (17).
In relation to glycerol produced in the lipolytic process, most of it is released into the bloodstream and mainly functions as substrates for glucose production in liver gluconeogenesis (3). Recently, with the discovery of the presence glycerol kinase, an enzyme the converts glycerol into glycerol 3-phosphate, and its activity in adipocytes raised the hypothesis that part of the glycerol produced by lipolysis may be recycled into TAG still inside of this cell (18).

Lipolysis is a process that occurs under non-stimulated (basal conditions) or in conditions stimulated by hormones $(19,20)$. In basal conditions, HSL predominantly remains in the cytosol, whereas perilipin, ATGL and its co-activator, protein Abhd5 (abhydrolase domain-containing 5), also called CGI-58 (comparative gene identification 58), are found on the surface of the lipid droplet (7). ATGL is highly specific for TAG hydrolysis, and it is responsible for large part of the lipolytic activity found both in basal and stimulated conditions. However, in basal conditions, the co-activator CGI-58 remains bound to perilipin (forming an inactive complex), that limits the hydrolytic activity of lipase $(9,19)$.

In periods of fasting or high energy demands, the adipocyte is stimulate by hormones, mainly by the action of catecholamines, leading to a series of intracellular reactions that culminates in the activation of protein kinase $A$ and $\mathrm{G}(\mathrm{PKA} / \mathrm{PKG})$, which promotes the phosphorylation of HSL and perilipin $(19,21,22)$. After phosphorylation, HSL is translocated into the lipid droplet and acts together with ATGL, accelerating the lipolytic process. Together, ATGL and HSL are responsible for about $95 \%$ of the hydrolysis of TAG (23). Similarly, after the phosphorylation of perilipin, the position of this compound on the lipid droplet changes, making TAG hydrolysis by the lipases easier. With the phosphorylation of perilipin, CGI-58 is released and binds to ATGL, forming an active complex and steeply increasing the action of this lipase $(13,19,22)$.

HSL is more specific for DAG hydrolysis, and may also hydrolyze TAG and MAG, with lower efficiency, though. MGL, on the other hand, is located both in the cytosol of the cell and on the surface of the lipid droplet, and shows specificity for MAG hydrolysis, catalyzing the final step of lipolysis, that is, the conversion of MAG into glycerol and a free fatty acid $(13,19)$ (Figure 2).

ATGL shows strong specificity and/or selectivity for the hydrolysis of the long-chain fatty acid esters (16 
or 18 carbons) found in position sn 2 of the glycerol backbone, which mainly includes the hydrolysis of palmitoleic acid followed by palmitic and stearic acid. The main product of this reaction is $1,3-\mathrm{DAG}$, the preferential substrate of the lipogenic enzyme diacylglycerol acyltransferase 2 (DGAT2). This suggests that the enzymes ATGL and DGAT act in a coordinated fashion during the TAG hydrolysis/reesterification cycle. On the other hand, after binding to CGI-58, ATGL starts to hydrolyze fatty acids also found in position snl, releasing 2,3-DAG, which is the preferential substrate of HSL (24).

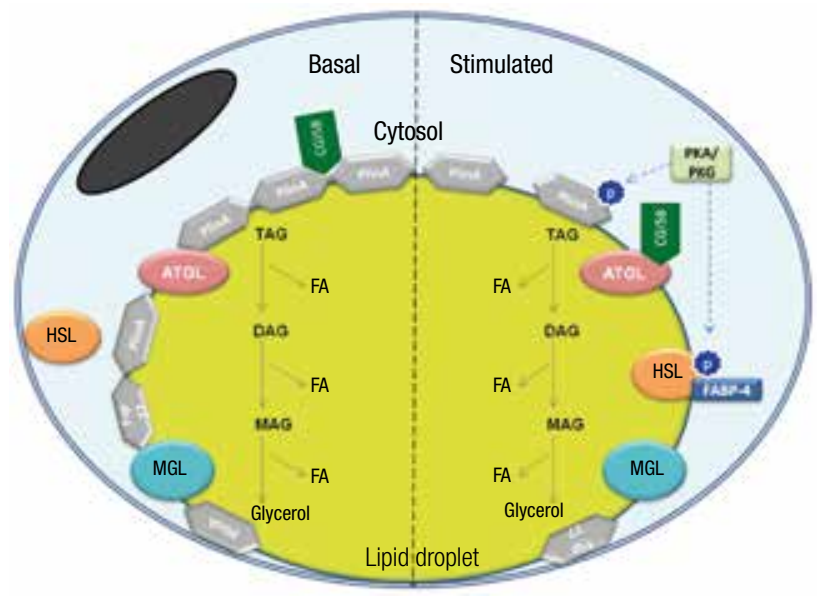

Figure 2. Non-stimulated (basal) and stimulated lipolysis in white adipocytes.

The lipid droplet is surrounded by a monolayer of phospholipids with different proteins anchored to it: PlinA and Fsp27/Cidec. In basal status (non-stimulated), ATGL, perilipin and CGI-58 form a complex on the surface of the lipid droplet. HL and FABP4 are in the cytosol. In the stimulated status, HSL is phosphorylated by a kinase (PKA and/or PKG) and, in its active form, migrates to the surface of the lipid droplet. PlinA is also phosphorylated and undergoes a change in structure. Because of that, $\mathrm{CGl}-58$ is released from PlinA and binds to ATGL, forming an active complex and increasing TAG hydrolysis. DAGs are then transformed into MAG by the action of HSL. The lipolytic process ends with MAG hydrolysis by MGL, with the release of three FA and one glycerol molecule. FA: fatty acid; ATGL: adipose triglyceride lipase; DAG: diacylglycerol; CGI-58: comparative gene identification 58; FABP4: fatty acid binding protein 4; Fsp27: fat-specific protein 27; HSL: hormone-sensitive lipase; MGL: monoacylglycerol lipase; MAG: monoacylglycerol; PlinA: perilipin 1A; PKA: protein kinase A; PKG: protein kinase G; TAG: triacylglycerol.

\section{ADIPOSE TRIGLYCERIDE LIPASE (ATGL) - FUNCTION AND REGULATION}

In 2004, three independent research groups characterized a new lipase that received different names, among them, ATGL, desnutrin, calcium-independent phospholipase $\mathrm{A} 2$ or patatin-like phospholipase domain-containing protein Al to A9 (PNPLAl-9) (14-16). ATGL, which has a molecular weight of $54 \mathrm{kDa}$, is found predominantly in white or brown adipose tissue, but is also seen in the testicles, pancreatic islets, cardiac muscle, and skeletal muscle (9). ATGL is nowadays considered the main enzyme responsible for TAG hydrolysis, and is mainly located in the lipid droplet of adipocytes ( 1,7 $9,13)$. Therefore, its hydrolytic activity on TAG takes place independent of the protein barrier that involves the lipid droplet, making this lipase very important in basal lipolysis. However, its activity is significantly increased by the interaction with protein CGI-58, its co-activator, in hormone-stimulated lipolysis $(7,22)$.

Studies in animal and cell cultures with reduction or overexpression of ATGL were carried out to characterize and describe biological functions of these enzymes and their participation in lipid homeostasis. In these studies, genetically modified ATGL-deficient mice showed reduced basal and stimulated lipolysis in adipocytes isolated from gonadal adipose tissue, as evidenced by reduced release of free fatty acids and glycerol in the incubation medium. Besides, these animals presented reduced concentration of free fatty acids in the plasma, both in fasting and not (25). Similarly, reduced ATGL expression induced by RNA interference in an experimental model of human adipocytes, hMADS cells, led to a decrease in basal lipolysis and halted lipolysis stimulated by forskolin (26).

The main phenotypical consequence of ATGL reduction is the massive accumulation of TAG in adipocytes and other tissues, favoring obesity development and other metabolic complications $(19,25)$. Still, this reduced TAG hydrolysis negatively affects thermoregulation in these animals, due to the reduced offer of free fatty acids for mitochondrial respiration. Therefore, mice with lower expression of ATGL show severe hypothermia when exposed to cold environments for five hours (25).

In the cardiac muscle, for example, the lack of ATGL leads to an increase of up to 20 times TAG content, causing cardiac failure followed by premature death. Reestablishment of ATGL expression only in the heart reverts the accumulation of TAG and associated cardiac failure (27). Besides, ATGL-deficient mice develop micro- and macro-endothelial dysfunctions that are reverted after treatment with agonists of nuclear peroxisome proliferator-activated receptors (PPAR) type $(\alpha)(28)$.

As for the lipid profile, ATGL-deficient animals show reduced plasma concentration of free fatty acids 
TAG, $\beta$-hidroxybutirate, total cholesterol, very low density lipoprotein (VLDL) and high density lipoprotein (HDL) (19). In pancreatic islets, lipolysis promoted by ATGL is essential to generate signaling lipid molecules that modulate positively glucose-stimulated insulin secretion (GSIS), an effect that depends on PPAR type delta $(\delta)$ (29). Different from the deficiency, ATGL overexpression is associated with increased basal and stimulated lipolysis (19), fatty acid oxidation, and reduced TAG deposition and adipocyte size (30).

Several factors have been identified in the regulation of gene expression and ATGL hydrolytic activity. The levels of messenger RNA (RNAm) of this enzyme are elevated, for example, by the action of PPAR agonists type $\alpha$ and $\gamma$, glucocorticoids, AMP-activated protein kinase (AMPK), and fasting; and are reduced by insulin, activation of mTOR complex 1 , and food intake (17,31-35).

Eight ATGL phosphorylation sites, including serine 406 and 430 have been recently described $(32,36,37)$. Although the importance of these phosphorylations for efficient ATGL activity has not been completely elucidated, it was already demonstrated that AMPK phosphorylates ATGL at serine 406, increasing its activity (32). Pagnon and cols. observed that phosphorylation at serine 406 of ATGL is also a PKA target, confirming that $\mathrm{B}$-adrenergic/catecholamine activation is capable of positive modulation of this lipase, accelerating lipolysis (36), as previously demonstrated for HSL. On the other hand, Xie and cols., in 2014, identified that ATGL phosphorylation at threonine 372 modulates the activity of the lipase negatively, hindering its location in the lipid droplet of the adipocytes (37).

Evidence also indicates that ATGL activity is modified by its interaction with other proteins, such as CGI58 , perilipin (previously described) and G0/Gl switch gene 2 (G0S2). G0S2, a protein that acts in the transition from G0 to $\mathrm{Gl}$ in the cell cycle, is located in adipocyte lipid droplet, cytoplasm, endoplasmic reticle and mitochondria. In the lipid droplet, G0S2 inhibits ATGL action by physical interaction of its $\mathrm{N}$-terminal with the N-terminal patatine domain of ATGL (38).

Additionally, fat-specific Protein 27 (FSP27), also found in the lipid droplet, interacts with ATGL reducing its activity and, consequently, decreasing lipolysis (39). Another important ATGL regulation mechanism involves the expression and control of the proteins that transport ATGL from the endoplasmic reticle to the lipid droplet, such as ADP-ribosylation factor 1, GTB- binding protein $\mathrm{l}$ (SARl), and golgi-brefeldin A resistance factor (GBF-1) (40).

All these findings point out to ATGL as the main enzyme responsible for TAG hydrolysis. Still, many studies are being carried out in order to clarify the mechanisms that regulate the activity of this lipase, as well as its role in physiological process in different tissues.

\section{HORMONE-SENSITIVE LIPASE (HSL) - FUNCTION AND REGULATION}

In the beginning of the 1960s, it was observed that lipolytic activity in the adipose tissue was induced by hormone stimulation. A classical study was published by Dr. Steimberg's group (41) describing HSL and MGL. At that moment, HSL, a protein of $81 \mathrm{kDa}$, was considered the main lipase of adipocytes, and the only one involved in TAG hydrolysis, as ATGL had not been discovered yet. HSL has at least five phosphorylation sites, from which serine 660 and 663 seem to be particularly important for hydrolytic activity (42). Haemmerle and cols., using HSL-deficient mice, observed that animals did not accumulate TAG in adipose and other tissues. Differently, they accumulated large amounts of DAG; determining, therefore, the high specificity of HSL in DAG hydrolysis (43).

HSL-deficient mice showed reduced hormonestimulated lipolysis (43). Similarly, mice that overexpress this lipase show normal basal lipolytic activity, and increased stimulated lipolysis (44).

The regulation of this lipase activity is highly influenced by hormones. Among them, catecholamines (released by the adrenal medulla or by direct sympathetic innervation of the adipose tissue), the atrial natriuretic peptide (ANP), and growth hormone (GH) are important activators. Insulin is an important inhibitor of HSL (17). Catecholamines bind to $\beta$-adrenergic receptors that bind to stimulatory protein $\mathrm{G}$, activating adenylate cyclase, which increases the levels of AMPc and, consequently, activates PKA. On its turn, PKA phosphorylates HSL and perilipin. ANP stimulates lipolysis by PKG activation $(9,45)$. On the other hand, the antilipolytic effect of insulin is mediated by phosphodiesterase $3 \mathrm{~B}$ activation, which decreases AMPc and PKA activity, reducing HSL and perilipin phosphorylation (45). Insulin promotes an increase in protein FSP27 in the lipid droplet and increases adipocyte-specific phospholipase A2 (AdPLA), reducing adenylate cyclase ac- 
tivity and, consequently, reducing cytoplasmic levels of AMPc and PKA activity $(46,47)$.

Another known form of regulation of this enzyme is via FABP (fatty acid binding protein). FABP4 binds to HSL when it is phosphorylated, making it easier for fatty acid to be transported inside of the cell after lipolysis. However, FABP4 regulates its action as it promotes a negative feedback for HSL by transporting fatty acids and/or by affecting the dimerization of the enzyme in the lipid droplet (48).

\section{MONOACYLGLYCEROL LIPASE (MGL) - FUNCTION AND REGULATION}

MGL catalyzes the final step of TAG hydrolysis, that is, MAG hydrolysis into glycerol and fatty acid. Its molecular weight is $33 \mathrm{kDa}$ and it belongs to the serine hydrolase superfamily (41). Evidence shows that MGL hydrolyses specifically MAG, and affects TAG or DAG (49). At the cell level, MGL is located in the cytoplasm, in the plasma membrane and in the lipid droplet. It is widely expressed in the WAT, lungs, liver, kidneys, testicles, brain, and heart (49). Animals deficient in MGL showed to be protected from the development of glucose intolerance and insulin resistance; reduced MGL activity is partially reverted by HSL (50). The MGL-deficient phenotype in animals is partially influenced by its effect on the central nervous system and in the endocannabinoid system (50). It is still unclear if MGL activity is influenced by cell energy status or hormones. In spite of this, it was demonstrated that treating rats with rosiglitazone, a PPAR $\gamma$ agonist $(15 \mathrm{mg} /$ $\mathrm{kg}$ for 7 days), positively regulates lipolysis and RNAm transcription of this lipase in WAT (51).

\section{PROTEINS ASSOCIATED WITH THE LIPID DROPLET}

Proteins associated with the lipid droplet, besides protecting cell organelles from the cytotoxicity caused by the lipids, have a fundamental role in lipolysis regulation $(8,52)$. These proteins belong the PAT family, which includes perilipins 1 to 5 . Perilipin 1 has three isoforms: perilipin $\mathrm{lA}$, perilipin $\mathrm{IB}$, and perilipin $\mathrm{IC}$, with perilipin IA as the main isoform found in WAT. Perilipin 5, or OXPAT, is mainly expressed in tissues of high oxidative capacity, such a brown adipose tissue and skeletal muscle, whereas perilipin 2 and perilipin 3 are found in the adipose tissue and liver $(7,53)$. As described throughout the text, when it is phosphorylated by PKA or PKG, perilipin undergoes a change in position on the lipid droplet, making HSL interaction with the droplet easier. Besides, it releases CGI-58, which binds to ATGL. A reduction in the expression of perilipin 1 leads to increased lipolysis, attributed to greater HSL activity and to the ATGL/CGI-58 interaction (54). On the other hand, perilipin 1 overexpression leads to reduced stimulated lipolysis without changes in basal lipolysis (55).

Recently, new proteins associated with the lipid droplet have been described, such as the CIDE family (cell death-inducing DNA fragmentation factor-alike effector). The CIDE family has three members: Cidea, Cideb, and Cidec/Fsp27 (7). Cidea and Cideb are found in brown adipose tissue and the liver, but are not expressed in the WAT. On the other hand, Cidec is highly expressed in white and brown adipose tissue. Its deficiency leads to increased basal lipolysis and does not change stimulated lipolysis levels $(39,56)$.

\section{PEROXISOME PROLIFERATOR-ACTIVATED RECEPTORS (PPARS) AND LIPOLYSIS}

PPARs $\alpha, \gamma$, and $\delta$ are members of the large family of nuclear receptors that act as transcription factors, controlling genes the have fundamental roles in the regulation of lipid metabolism, inflammation, and cell growth and differentiation (57). These nuclear receptors are known as lipid sensors, and have their production and activity highly influenced by nutritional status. Among their important effects in the adipocytes, PPAR $\gamma$ and $\alpha$ have also been described to positively modulate lipolysis $(51,58)$.

PPAR $\gamma$ activation with rosiglitazone, for example, stimulates lipolysis and increases RNAm expression of ATGL e MGL in vivo (51). Corroborating this study, Kershaw and cols. demonstrated that PPAR $\gamma$ positively regulates RNAm and the protein content of ATGL in mature adipocytes, in vitro and in vivo, suggesting that ATGL is a mediator of the PPAR $\gamma$ effects in lipid metabolism (33).

Similarly, treatment of rats with a PPAR $\alpha$ agonist, fenofibrate, induces a significant increase of 30\% in lipolysis speed in the presence of insulin in WAT (59). Besides, an endogenous elevation of the lipid oleoylethanolamine in the small intestine stimulates lipolysis and fatty acid oxidation in epididymis adipose tissue by mechanisms that involve the activation of PPAR $\alpha$ (34). In this context, our group has recently demonstrated 
that adipocyte treatment in vivo and in vitro with palmitoleic acid increases lipolysis, under basal conditions and after stimulation with isoproterenol, and the protein content of ATGL and HSL phosphorylated at serine 660 by a PPAR $\alpha$-dependent mechanism (58). Overall, these results determine PPAR $\gamma$ and PPAR $\alpha$ as important regulators of the lipolysis in the white adipose tissue, besides their known roles as positive modulators of adipogenesis and $\beta$-oxidation/mitochondrial function, respectively (57).

On the other hand, fatty acids derived from lipolysis are important PPAR activators. According to Zechner and cols., plasma fatty acids, when entering cells, do not bind directly to PPAR (17). A fatty acid esterification and hydrolysis cycle is necessary for these molecules to act as PPAR ligands $(17,60)$. This hypothesis was proven by the reduced lipolytic activity found in ATGL-deficient animals, which causes important reduction in PPAR $\alpha$ signaling. Consequently, oxidation of substrates of several tissues and cell types, such as liver, macrophages, and brown adipose tissue, is reduced $(17,32,61,62)$. HSL deficiency also moderately reduces PPAR $\alpha$ signaling (60). Besides, ATGL deficiency leads to reduced PPAR $\alpha$ activation in the heart and vascular endothelium, and PPAR $\delta$ in the pancreas, deeply compromising the physiological function of these tissues $(28,29,60)$.

\section{AMP-ACTIVATED PROTEIN KINASE (AMPK) AND LIPOLYSIS}

AMPK is an important molecule in the regulation of cell energy balance. It is sensitive to nutritional status and responds to reduction in the ATP/AMP ratio. This molecule is able to phosphorylate and regulate proteins related to energy metabolism, decreasing the anabolic pathways (ATP consumption) and increasing catabolic ones (ATP generation - such as the increase in glucose capture and oxidation of fatty acids and glucose) $(32,35,63,64)$.

Studies revealed important effects of AMPK in the WAT metabolism, including lipolysis regulation $(32,63,64)$. A previous study found that acute AMPK activation with AICAR (5-amino- $1-\beta$-D-ribofuranosylimidazole-4-carboxamide), an AMP analogue, promotes inhibitory phosphorylation of HSL at serine 565 (65). However, as demonstrated by Ahmadian and cols., AMPK activation with AICAR leads to phosphorylation of ATGL at serine 406, expressively in- creasing the activity of this lipase and, therefore, lipolysis in HEK293 cells and 3T3-Ll adipocytes (32). In another study, prolonged activation of AMPK induced by the treatment of epididymis adipocytes in vitro with $\operatorname{AICAR}(0.5 \mathrm{mM})$ for 15 hours, or rats treated with a single intraperitoneal injection of AICAR $(0.7 \mathrm{~g} /$ $\mathrm{kg}$ ) showed increased lipolysis, ATGL protein content, and gene expression of PPAR $\alpha, \operatorname{PPAR} \delta$, and PGC- $1 \alpha$ (64). Additionally, chronic activation of AMPK in the adipose tissue induced remodeling of the metabolism of these cells, potentiating catabolic pathways of ATP production instead of routes of energy storage and ATP consumption (64).

AMPK effect in the activation of lipolysis in WAT, increasing the offer of fatty acids and glycerol to be used as energy substrates in different tissues may be another mechanism by which this kinase favors the increased production of ATP to reestablish energy homeostasis. This phenomenon is the proof of the important contribution of lipolytic activity and lipases in the control of the energy metabolism.

\section{FINAL REMARKS}

Since the discovery of ATGL in 2004, a large number of studies have been carried out leading to wide progress in lipolysis characterization, besides revealing a growing number of enzymes, kinases, and regulatory proteins, recently.

It is clear, nowadays, that lipolysis is not only a process that supplies energy substrates for ATP generation in different tissues, but that lipid products (fatty acids and their derivatives) have essential roles in multiple systemic and intracellular signaling pathways, including, for example, PPARs $(17,52)$. These roles of lipolysis are not restricted to the WAT, as proven by severe damage found in the pancreas, liver, heart and vascular endothelium associated with reduced lipolysis. Normal lipolytic activity seems to be crucial for energy homeostasis in different tissues.

On the other hand, excessive mobilization of fatty acids found in lipolysis was previously associated with high plasma concentrations of fatty acids, dyslipidemias, and insulin resistance (12). Because of that, prescription of drugs that reduce lipolytic activity has been a therapeutic strategy in the control of these diseases, although there are objections to the efficacy of these drugs, such as nicotinic acid and its analogue, Acipimox $^{\circledR}(13,66)$. 
Curiously, studies have demonstrated that animals that show increased lipolytic activity have reduced adipose mass without, however, elevation of fatty acids serum levels. This fact is due to the fact that these animals show greater $\beta$-oxidation and reesterification of fatty acids into TAG (TAG-fatty acid cycle or futile cycle) inside the adipocytes $(58,67-69)$. These data reveal that drugs/substances that are capable of activating lipolysis in white adipocytes are promising therapeutic strategies for the treatment of obesity.

Evidently, there are ongoing studies to increase the knowledge on this lipolytic machinery and its regulation, as well as on the importance of lipolysis on multiple signaling pathways of body tissues.

Acknowledgements: we would like to thank Dr. William T. Festuccia for his comments and suggestions for this article.

Disclosure: no potential conflict of interest relevant to this article was reported.

\section{REFERENCES}

1. Lafontan M. Advances in adipose tissue metabolism. Int $\mathrm{J}$ Obes (Lond). 2008;32 Suppl 7:S39-51.

2. Frayn KN. Adipose tissue as a buffer for daily lipid flux. Diabetologia. 2002;45(9):1201-10.

3. Nelson D, Cox M. Lehninger - Princípios de Bioquímica. 3 ed. São Paulo: Savier; 2003. 779-824 p.

4. OttoTC, Lane MD. Adipose development: from stem cell to adipocyte. Crit Rev Biochem Mol Biol. 2005;40(4):229-42.

5. Castro-Chavez F, Yechoor VK, Saha PK, Martinez-Botas J, Wooten EC, Sharma S, et al. Coordinated upregulation of oxidative pathways and downregulation of lipid biosynthesis underlie obesity resistance in perilipin knockout mice: a microarray gene expression profile. Diabetes. 2003;52(11):2666-74.

6. Londos C, Brasaemle DL, Schultz CJ, Segrest JP, Kimmel AR. Perilipins, ADRP, and other proteins that associate with intracelIular neutral lipid droplets in animal cells. Semin Cell Dev Biol. 1999;10(1):51-8.

7. Girousse A, Langin D. Adipocyte lipases and lipid droplet-associated proteins: insight from transgenic mouse models. Int $\mathrm{J}$ Obes (Lond). 2012;36(4):581-94.

8. Brasaemle DL. Thematic review series: adipocyte biology. The perilipin family of structural lipid droplet proteins: stabilization of lipid droplets and control of lipolysis. J Lipid Res. 2007;48(12):2547-59.

9. Lafontan M, Langin D. Lipolysis and lipid mobilization in human adipose tissue. Prog Lipid Res. 2009;48(5):275-97.

10. Large V, Peroni O, Letexier D, Ray H, Beylot M. Metabolism of lipids in human white adipocyte. Diabetes Metab. 2004;30(4):294-309.

11. Guyenet SJ, Schwartz MW. Clinical review: regulation of food intake, energy balance, and body fat mass: implications for the pathogenesis and treatment of obesity. J Clin Endocrinol Metab. 2012;97(3):745-55.

12. Bergman $\mathrm{RN}$, Ader M. Free fatty acids and pathogenesis of type 2 diabetes mellitus. Trends Endocrinol Metab. 2000;11(9):351-6.

13. Ahmadian M, Wang $Y$, Sul HS. Lipolysis in adipocytes. Int J Biochem Cell Biol. 2010;42(5):555-9.
14. Zimmermann R, Strauss JG, Haemmerle G, Schoiswohl G, Birner-Gruenberger $\mathrm{R}$, Riederer $\mathrm{M}$, et al. Fat mobilization in adipose tissue is promoted by adipose triglyceride lipase. Science. 2004;306(5700):1383-6.

15. Jenkins CM, Mancuso DJ, Yan W, Sims HF, Gibson B, Gross RW. Identification, cloning, expression, and purification of three novel human calcium-independent phospholipase A2 family members possessing triacylglycerol lipase and acylglycerol transacylase activities. J Biol Chem. 2004;279(47):48968-75.

16. Villena JA, Roy S, Sarkadi-Nagy E, Kim KH, Sul HS. Desnutrin, an adipocyte gene encoding a novel patatin domain-containing protein, is induced by fasting and glucocorticoids: ectopic expression of desnutrin increases triglyceride hydrolysis. J Biol Chem. 2004;279(45):47066-75.

17. Zechner R, Zimmermann R, Eichmann TO, Kohlwein SD, Haemmerle G, Lass A, et al. FAT SIGNALS--lipases and lipolysis in lipid metabolism and signaling. Cell Metab. 2012;15(3):279-91.

18. Guan HP, LiY, Jensen MV, Newgard CB, Steppan CM, Lazar MA. A futile metabolic cycle activated in adipocytes by antidiabetic agents. Nat Med. 2002;8(10):1122-8.

19. Bézaire $V$, Langin D. Regulation of adipose tissue lipolysis revisited. Proc Nutr Soc. 2009;68(4):350-60.

20. Duncan RE, Ahmadian M, Jaworski K, Sarkadi-Nagy E, Sul HS. Regulation of lipolysis in adipocytes. Annu Rev Nutr. 2007;27:79-101.

21. Mauriège $P$, De Pergola $G$, Berlan $M$, Lafontan $M$. Human fat cell beta-adrenergic receptors: beta-agonist-dependent lipolytic responses and characterization of beta-adrenergic binding sites on human fat cell membranes with highly selective beta 1-antagonists. J Lipid Res. 1988;29(5):587-601.

22. Yamaguchi T, Omatsu N, Matsushita S, Osumi T. CGI-58 interacts with perilipin and is localized to lipid droplets. Possible involvement of CGI-58 mislocalization in Chanarin-Dorfman syndrome. J Biol Chem. 2004;279(29):30490-7.

23. Schweiger M, Schreiber R, Haemmerle G, Lass A, Fledelius C, Jacobsen $\mathrm{P}$, et al. Adipose triglyceride lipase and hormone-sensitive lipase are the major enzymes in adipose tissue triacylglycerol catabolism. J Biol Chem. 2006;281(52):40236-41.

24. EichmannTO, Kumari M, Haas JT, Farese RV, Zimmermann R, Lass $A$, et al. Studies on the substrate and stereo/regioselectivity of adipose triglyceride lipase, hormone-sensitive lipase, and diacylglycerol-O-acyltransferases. J Biol Chem. 2012;287(49):41446-57.

25. Haemmerle G, Lass A, Zimmermann R, Gorkiewicz G, Meyer C, Rozman J, et al. Defective lipolysis and altered energy metabolism in mice lacking adipose triglyceride lipase. Science. 2006;312(5774):734-7.

26. Bezaire V, Mairal A, Ribet C, Lefort C, Girousse A, Jocken J, et al. Contribution of adipose triglyceride lipase and hormonesensitive lipase to lipolysis in hMADS adipocytes. J Biol Chem. 2009;284(27):18282-91.

27. Schoiswohl G, Schweiger M, Schreiber R, Gorkiewicz G, PreissLandl K, Taschler U, et al. Adipose triglyceride lipase plays a key role in the supply of the working muscle with fatty acids. J Lipid Res. 2010;51(3):490-9.

28. Schrammel A, Mussbacher M, Wölkart G, Stessel H, Pail K, Winkler $\mathrm{S}$, et al. Endothelial dysfunction in adipose triglyceride lipase deficiency. Biochim Biophys Acta. 2014;1841(6):906-17.

29. Tang T, Abbott MJ, Ahmadian M, Lopes AB, Wang Y, Sul HS. Desnutrin/ATGL activates PPAR $\delta$ to promote mitochondrial function for insulin secretion in islet $\beta$ cells. Cell Metab. 2013;18(6):883-95.

30. Smirnova E, Goldberg EB, Makarova KS, Lin L, Brown WJ, Jackson CL. ATGL has a key role in lipid droplet/adiposome degradation in mammalian cells. EMBO Rep. 2006;7(1):106-13.

31. Chakrabarti P, English T, Shi J, Smas CM, Kandror KV. Mammalian target of rapamycin complex 1 suppresses lipolysis, stimulates lipogenesis, and promotes fat storage. Diabetes. 2010;59(4):775-81.

32. Ahmadian M, Abbott MJ, Tang T, Hudak CS, Kim Y, Bruss M, et al. Desnutrin/ATGL is regulated by AMPK and is required for a brown adipose phenotype. Cell Metab. 2011;13(6):739-48. 
33. Kershaw EE, Schupp M, Guan HP, Gardner NP, Lazar MA, Flier JS. PPARgamma regulates adipose triglyceride lipase in adipocytes in vitro and in vivo. Am J Physiol Endocrinol Metab. 2007;293(6):E1736-45.

34. Guzmán M, Lo Verme J, Fu J, Oveisi F, Blázquez C, Piomelli D. Oleoylethanolamide stimulates lipolysis by activating the nuclear receptor peroxisome proliferator-activated receptor alpha (PPARalpha). J Biol Chem. 2004;279(27):27849-54.

35. Wu X, Motoshima H, Mahadev K, Stalker TJ, Scalia R, Goldstein BJ. Involvement of AMP-activated protein kinase in glucose uptake stimulated by the globular domain of adiponectin in primary rat adipocytes. Diabetes. 2003;52(6):1355-63.

36. Pagnon J, Matzaris M, Stark R, Meex RC, Macaulay SL, Brown $W$, et al. Identification and functional characterization of protein kinase A phosphorylation sites in the major lipolytic protein, adipose triglyceride lipase. Endocrinology. 2012;153(9):4278-89.

37. Xie $X$, Langlais $P$, Zhang $X$, Heckmann BL, Saarinen AM, Mandarino $L J$, et al. Identification of a novel phosphorylation site in adipose triglyceride lipase as a regulator of lipid droplet localization. Am J Physiol Endocrinol Metab. 2014;306(12):E1449-59.

38. Lu X, Yang X, Liu J. Differential control of ATGL-mediated lipid droplet degradation by $\mathrm{CGI}-58$ and G0S2. Cell Cycle. 2010;9(14):2719-25.

39. GrahnTH, Kaur R, Yin J, Schweiger M, Sharma VM, Lee MJ, et al. Fat-specific Protein 27 (FSP27) Interacts with Adipose Triglyceride Lipase (ATGL) to Regulate Lipolysis and Insulin Sensitivity in Human Adipocytes. J Biol Chem. 2014;289(17):12029-39.

40. Ellong EN, Soni KG, Bui QT, Sougrat R, Golinelli-Cohen MP, Jackson CL. Interaction between the triglyceride lipase ATGL and the Arf1 activator GBF1. PLoS One. 2011;6(7):e21889.

41. Vaughan M. The production and release of glycerol by adipose tissue incubated in vitro. J Biol Chem. 1962;237:3354-8.

42. Anthonsen MW, Rönnstrand L, Wernstedt C, Degerman E, Holm C. Identification of novel phosphorylation sites in hormonesensitive lipase that are phosphorylated in response to isoproterenol and govern activation properties in vitro. $\mathrm{J}$ Biol Chem. 1998;273(1):215-21.

43. Haemmerle G, Zimmermann R, Hayn M, Theussl C, Waeg G, Wagner $E$, et al. Hormone-sensitive lipase deficiency in mice causes diglyceride accumulation in adipose tissue, muscle, and testis. J Biol Chem. 2002;277(7):4806-15.

44. Lucas S, Tavernier G, Tiraby C, Mairal A, Langin D. Expression of human hormone-sensitive lipase in white adipose tissue of transgenic mice increases lipase activity but does not enhance in vitro lipolysis. J Lipid Res. 2003;44(1):154-63.

45. Jaworski K, Sarkadi-Nagy E, Duncan RE, Ahmadian M, Sul HS. Regulation of triglyceride metabolism. IV. Hormonal regulation of lipolysis in adipose tissue. Am J Physiol Gastrointest Liver Physiol. 2007;293(1):G1-4.

46. Duncan RE, Sarkadi-Nagy E, Jaworski K, Ahmadian M, Sul HS. Identification and functional characterization of adipose-specific phospholipase A2 (AdPLA). J Biol Chem. 2008;283(37):25428-36.

47. Czech MP, Tencerova M, Pedersen DJ, Aouadi M. Insulin signalling mechanisms for triacylglycerol storage. Diabetologia. 2013;56(5):949-64.

48. Smith AJ, Thompson BR, Sanders MA, Bernlohr DA. Interaction of the adipocyte fatty acid-binding protein with the hormonesensitive lipase: regulation by fatty acids and phosphorylation. $J$ Biol Chem. 2007;282(44):32424-32.

49. Karlsson M, Contreras JA, Hellman U, Tornqvist $H$, Holm C. cDNA cloning, tissue distribution, and identification of the catalytic triad of monoglyceride lipase. Evolutionary relationship to esterases, lysophospholipases, and haloperoxidases. J Biol Chem. 1997;272(43):27218-23.

50. Taschler U, Radner FP, Heier C, Schreiber R, Schweiger M, Schoiswohl G, et al. Monoglyceride lipase deficiency in mice impairs lipolysis and attenuates diet-induced insulin resistance. J Biol Chem. 2011;286(20):17467-77.
51. Festuccia WT, Laplante M, Berthiaume M, Gélinas Y, Deshaies Y. PPARgamma agonism increases rat adipose tissue lipolysis, expression of glyceride lipases, and the response of lipolysis to hormonal control. Diabetologia. 2006;49(10):2427-36.

52. NielsenTS, Jessen N, Jørgensen JO, Møller N, Lund S. Dissecting adipose tissue lipolysis: molecular regulation and implications for metabolic disease. J Mol Endocrinol. 2014;52(3):R199-222.

53. Kimmel AR, Brasaemle DL, McAndrews-Hill M, Sztalryd C, Londos C. Adoption of PERILIPIN as a unifying nomenclature for the mammalian PAT-family of intracellular lipid storage droplet proteins. J Lipid Res. 2010;51(3):468-71.

54. Martinez-Botas J, Anderson JB, Tessier D, Lapillonne A, Chang BH, Quast MJ, et al. Absence of perilipin results in leanness and reverses obesity in Lepr(db/db) mice. Nat Genet. 2000;26(4):474-9.

55. Miyoshi H, Souza SC, Endo M, Sawada T, Perfield JW, Shimizu $C$, et al. Perilipin overexpression in mice protects against dietinduced obesity. J Lipid Res. 2010;51(5):975-82.

56. Toh SY, Gong J, Du G, Li JZ, Yang S, Ye J, et al. Up-regulation of mitochondrial activity and acquirement of brown adipose tissuelike property in the white adipose tissue of fsp27 deficient mice. PLoS One. 2008;3(8):e2890.

57. Poulsen L, Siersbæk M, Mandrup S. PPARs: fatty acid sensors controlling metabolism. Semin Cell Dev Biol. 2012;23(6):631-9.

58. Bolsoni-Lopes A, Festuccia WT, FariasTS, Chimin P, Torres-Leal FL, Derogis PB, et al. Palmitoleic acid ( $n-7)$ increases white adipocyte lipolysis and lipase content in a PPAR囚-dependent manner. Am J Physiol Endocrinol Metab. 2013;305(9):E1093-102.

59. Ferreira AV, Parreira GG, Green A, Botion LM. Effects of fenofibrate on lipid metabolism in adipose tissue of rats. Metabolism. 2006;55(6):731-5.

60. Haemmerle G, Moustafa T, Woelkart G, Büttner S, Schmidt A, van de Weijer T, et al. ATGL-mediated fat catabolism regulates cardiac mitochondrial function via PPAR- $\triangle$ and PGC-1. Nat Med. 2011;17(9):1076-85.

61. Chandak PG, Radovic B, Aflaki E, Kolb D, Buchebner M, Fröhlich E, et al. Efficient phagocytosis requires triacylglycerol hydrolysis by adipose triglyceride lipase. J Biol Chem. 2010;285(26):20192-201.

62. Ong KT, Mashek MT, Bu SY, Greenberg AS, Mashek DG. Adipose triglyceride lipase is a major hepatic lipase that regulates triacylglycerol turnover and fatty acid signaling and partitioning. Hepatology. 2011;53(1):116-26.

63. Bijland S, Mancini SJ, Salt IP. Role of AMP-activated protein kinase in adipose tissue metabolism and inflammation. Clin Sci (Lond). 2013;124(8):491-507.

64. Gaidhu MP, Fediuc S, Anthony NM, So M, Mirpourian M, Perry RL, et al. Prolonged AICAR-induced AMP-kinase activation promotes energy dissipation in white adipocytes: novel mechanisms integrating HSL and ATGL. J Lipid Res. 2009;50(4):704-15.

65. Su CL, Sztalryd C, Contreras JA, Holm C, Kimmel AR, Londos C. Mutational analysis of the hormone-sensitive lipase translocation reaction in adipocytes. J Biol Chem. 2003;278(44):43615-9.

66. Dhalla AK, Chisholm JW, Reaven GM, Belardinelli L. A1 adenosine receptor: role in diabetes and obesity. Handb Exp Pharmacol. 2009(193):271-95.

67. Ahmadian M, Duncan RE, Varady KA, Frasson D, Hellerstein MK, Birkenfeld AL, et al. Adipose overexpression of desnutrin promotes fatty acid use and attenuates diet-induced obesity. Diabetes. 2009;58(4):855-66.

68. Jaworski K, Ahmadian M, Duncan RE, Sarkadi-Nagy E, Varady $K A$, Hellerstein MK, et al. AdPLA ablation increases lipolysis and prevents obesity induced by high-fat feeding or leptin deficiency. Nat Med. 2009;15(2):159-68.

69. Saha PK, Kojima H, Martinez-Botas J, Sunehag AL, Chan L. Metabolic adaptations in the absence of perilipin: increased betaoxidation and decreased hepatic glucose production associated with peripheral insulin resistance but normal glucose tolerance in perilipin-null mice. J Biol Chem. 2004;279(34):35150-8. 